Universidade Federal do Espírito Santo (UFES)

Centro de Ciências Jurídicas e Econômicas (CCJE)

Programa de Pós-Graduação em Administração (PPGAdm)

Revista Gestão \& Conexões

Management and Connections Journal

Volume 8, Número 3, Set./Dez. 2019

Vitória (ES)

Brasil

Setembro de 2019

(Publicada em 30 de Setembro de 2019) 
Copyright @ 2019 - Gestão \& Conexões

\section{Apresentação da Revista}

A Revista Gestão \& Conexões é uma publicação do Programa de Pós-Graduação em Administração (PPGAdm/CCJE/UFES). Sua missão é contribuir para a discussão interdisciplinar da gestão organizacional, através da divulgação de artigos (teóricos e empíricos) e notas bibliográficas, no campo da Administração, subsidiando as atividades acadêmicas e a ação administrativa em organizações públicas, privadas e/ou do terceiro setor.

Universidade Federal do Espírito Santo (UFES)

Reinaldo Centoducatte (Reitor)

Centro de Ciências Jurídicas e Econômicas (CCJE)

Rogério Naques Faleiros (Diretor)

Tárek Moysés Moussallém (Vice-Diretor)

Programa de Pós-Graduação em Administração (PPGAdm)

Priscilla Martins da Silva (Coordenadora)

Letícia Dias Fantinel (Coordenadora Adjunta)

Endereço para Correspondência

Universidade Federal do Espírito Santo

Centro de Ciências Jurídicas e Econômicas

Programa de Pós-Graduação em Administração

Revista Gestão \& Conexões

(UFES/CCJE/PPGAdm/REGEC)

Av. Fernando Ferrari, \# 514, Goiabeiras

29.075-910, Vitória (ES), Brasil

Telefone: +55 (27) 31455370

Endereço Eletrônico

<gestao.conexoes@gmail.com>

Home-page

<http://www.periodicos.ufes.br/ppgadm>

Editora-Chefe

Lucilaine Pascuci (Espírito Santo, UFES), Brasil

\section{Conselho Editorial}

Adelaide Maria de Souza Antunes (Rio de Janeiro, UFRJ/INPI), Brasil

Alexandre de Pádua Carrieri (Minas Gerais, UFMG), Brasil

Ana Paula Paes de Paula (Minas Gerais, UFMG), Brasil

Christiane Kleinübing Godoi (Santa Catarina, UNIVALI), Brasil

Eduardo Paes Barreto Davel (Bahia, UFBA), Brasil

Eduardo Pinheiro Gondim de Vasconcellos (São Paulo, FEA/USP), Brasil

Eduardo Raupp de Vargas (Brasília, UnB), Brasil

Hernán Eduardo Thomas (Bernal/Buenos Aires/Argentina, UNQ), Argentina

Jair Nascimento Santos (Bahia, UEFS), Brasil

José Henrique de Faria (Paraná, UFPR), Brasil

Josep Maria Blanch (UAB), Espanha

Leda Maria Caira Gitahy (São Paulo, DPCT/IG/UNICAMP), Brasil 
Liliana Rolfsen Petrilli Segnini (São Paulo, DECISE/FE/UNICAMP), Brasil Luís Paulo Bresciani (São Paulo, USCS), Brasil

Luiz Alex Silva Saraiva (Minas Gerais, UFMG), Brasil

Nadya Araujo Guimarães (São Paulo, FFLCH/USP), Brasil

Neusa Rolita Cavedon (Rio Grande do Sul, UFRGS), Brasil

Nilton Vargas (São Paulo, NEOLABOR, UNICAMP), Brasil

Omar Aktouf (Montréal, HEC), Canadá

Rafael Alcadipani da Silveira (São Paulo, EAESP/FGV), Brasil

Roberto Carlos Bernardes (São Paulo, FEI), Brasil

Rodrigo Bandeira-de-Mello (São Paulo, EAESP/FGV), Brasil

Tales Andreassi (São Paulo, EAESP/FGV), Brasil

Vera Lúcia Cançado (Minas Gerais, FPL), Brasil

Wilson Suzigan (São Paulo, DPCT/IG/UNICAMP), Brasil

\section{Corpo Editorial Científico}

Alfredo Rodrigues Leite da Silva (Espírito Santo, UFES), Brasil

Flávia Luciane Consoni (São Paulo, UNICAMP, CEBRAP), Brasil

Glicia Vieira dos Santos (Espírito Santo, UFES), Brasil

Marcos Paulo Valadares de Oliveira (Espírito Santo, UFES), Brasil

Identidade Visual

Eliza Gobira (Espírito Santo, SECOM/UFES, Brasil)

Capa

Equipe Editorial (Espírito Santo, UFES, Brasil)

Revisão e Normalização

Valéria Silva da Fonseca (Curitiba, Paraná, Brasil)

Diagramação e Formatação Final

Equipe Editorial (Espírito Santo, UFES), Brasil

Edição disponibilizada em 30 de setembro de 2019. 


\section{Catalogação na Fonte}

Gestão \& Conexões = Management and Connections Journal / Universidade Federal do Espírito Santo. Centro de Ciências Jurídicas e Econômicas. Programa de Pós-Graduação em Administração . - v. 8, n.3 (set./dez. 2019) - . - Vitória : PPGAdm, 2019-

Quadrimestral.

Resumo em português e inglês.

Disponível no Portal de Periódicos UFES em: <http://www.periodicos.ufes.br/ppgadm/>. Versão online ISSN 2317-5087.

1. Administração - Periódico. 2. Organizações, Subjetividades e Simbolismo - Periódico; 3. Tecnologias e Processos Organizacionais - Periódico; 4. Política e Gestão de Ciência, Tecnologia e Inovação - Periódico. I. Universidade Federal do Espírito Santo. Programa de Pós-Graduação em Administração.

CDU 658 